SAGA-HE-230

KEK-TH-1110

\title{
Ginsparg-Wilson Dirac operator in the monopole backgrounds on the fuzzy 2-sphere
}

\author{
Hajime Aokid1, Satoshi Iso 42 and Toshiharu Maedad \\ ${ }^{a}$ Department of Physics, Saga University, Saga 840-8502, Japan \\ ${ }^{b}$ Institute of Particle and Nuclear Studies, \\ High Energy Accelerator Research Organization (KEK) \\ Tsukuba 305-0801, Japan
}

\begin{abstract}
In the previous papers, we studied the 't Hooft-Polyakov (TP) monopole configurations in the $U(2)$ gauge theory on the fuzzy 2 -sphere, and showed that they have nonzero topological charges in the formalism based on the Ginsparg-Wilson (GW) relation. In this paper, we will show an index theorem in the TP monopole background, which is defined in the projected space, and provide a meaning of the projection operator. We also extend the index theorem to general configurations which do not satisfy the equation of motion, and show that the configuration space can be classified into the topological sectors. We further calculate the spectrum of the GW Dirac operator in the TP monopole backgrounds, and consider the index theorem in these cases.
\end{abstract}

\footnotetext{
${ }^{1}$ e-mail address: haoki@cc.saga-u.ac.jp

${ }^{2}$ e-mail address: satoshi.iso@kek.jp

${ }^{3}$ e-mail address: maeda@th.phys.saga-u.ac.jp
} 


\section{Introduction}

Matrix models are a promising candidate to formulate the superstring theory nonperturbatively [1, 2], where both spacetime and matter are described in terms of matrices, and noncommutative (NC) geometries [3] naturally appear [4, 5, 6]. One of the important subjects of the matrix model is a construction of configurations with nontrivial indices in finite NC geometries, since compactifications of extra dimensions with nontrivial indices can realize chiral gauge theories in our spacetime. Topologically nontrivial configurations in NC geometries were constructed by using algebraic K-theory and projective modules [7, 8, 9, 10, 11, 12].

In order to see their relation to indices of Dirac operators, a suitable framework will be the one where the chirality operator and the Dirac operator satisfy the Ginsparg-Wilson (GW) relation[13], since NC geometries on some compact manifolds have only finite degrees of freedom. The GW relation has been developed in the lattice gauge theory. Its explicit construction was given by the overlap Dirac operator [14] and the perfect action [15]. The exact chiral symmetry[16, 17] and the index theorem[15, 16] at a finite cutoff can be realized due to the GW relation.

In ref.[18], we have provided a general prescription to construct a GW Dirac operator with coupling to nonvanishing gauge field backgrounds on general finite NC geometries. As a concrete example we considered the fuzzy 2-sphere[19]1. Owing to the GW relation, an index theorem can be proved even for finite $\mathrm{NC}$ geometries. We have defined a topological charge, and showed that it takes only integer values, and becomes the Chern character in the commutative limit $[18,21,22,23]^{2}$.

\footnotetext{
${ }^{1}$ The GW Dirac operator on the fuzzy 2 -sphere for vanishing gauge field was constructed earlier in 20$]$.

2 The GW relation was implemented also on the NC torus by using the Neuberger's overlap Dirac operator in [24]. In [25], this GW Dirac operator was also derived from the general prescription [18] and the correct chiral anomaly was reproduced by using a topological method
} 
We then constructed the 't Hooft-Polyakov (TP) monopole configuration as a topologically nontrivial configuration [22, 23]. We showed that this configuration is a NC analogue of the commutative TP monopole by explicitly studying the form of the configuration. We then redefined the topological charge by inserting a projection operator, and showed that it reproduces the correct form of the topological charge in the commutative limit. We also showed that the topological charge takes the appropriate values for the TP monopole configurations. Furthermore, in [28], we presented a mechanism for the dynamical generation of a nontrivial index, by showing that the TP monopole configurations are stabler than the topologically trivial sector in the Yang-Mills-Chern-Simons matrix model [29, 30] 3 .

In this paper, we will prove an index theorem in the TP monopole backgrounds. The TP monopole configuration breaks the $S U(2)$ gauge symmetry down to $U(1)$, and the matter field in the fundamental representation of the $S U(2)$ gauge group has two components, corresponding to $+1 / 2$ and $-1 / 2$ electric charges of the unbroken $U(1)$ gauge group. Since these two components cancel the index and the chiral anomaly, we need to pick up one of them. The index with a projection operator to pick up one of the two components is shown to give the above topological charge introduced in ref. [22, 23, 28].

The index theorem can be extended to more general configurations which do not satisfy the equation of motion. By modifying the chirality operators and the GW Dirac operator in general gauge field configurations on the fuzzy 2-sphere, we propose a topological charge classifying configurations in spontaneously symmetry broken gauge theories. This topological charge is shown to become the 't Hooft's topological charge in the commutative limit. Since the $U(2)$ gauge theory on the fuzzy sphere is generally broken down to $U(1) \times U(1)$ gauge theory through Higgs mechanism, this generalization shows that the configuration space of gauge in [26]. The correct parity anomaly was reproduced in [27].

${ }^{3}$ The stability of these configurations are also investigated in papers $30,31,32,33,34,35$. 
fields on the fuzzy sphere can be classified into the topological sectors. We also discuss the validity of this classification by introducing the concept of admissibility condition, which was developed to investigate the topological structure in the lattice gauge theory.

We also calculate the spectrum of the GW Dirac operator in the TP monopole backgrounds 4 , and confirm the index theorem in these cases. The spectrum was also provided in [22]. We study the spectrum in more details and obtain the explicit forms of the eigenstates by using the GW relation. The largest eigenvalue states are shown to play an important role in the index theorem.

In section 2 we briefly review how to define the GW Dirac operator on the fuzzy 2-sphere and how to construct the TP monopole configurations. We then show the index theorem in the TP monopole backgrounds, and give an interpretation of the projection operator. We further extend the index theorem to general configurations. In section 3, we calculate the spectrum for the GW Dirac operator in the TP monopole backgrounds, and in section 4, we obtain the forms of the chiral zero-modes. Section 5 is devoted to conclusions and discussions. In appendix A, we calculate the spectrum for another type of Dirac operator $D_{\mathrm{GKP}}$, which is given in (2.7). In appendix B, we abtain the spectrum for the Dirac operator in the commutative theory.

\footnotetext{
${ }^{4}$ While we are preparing the manuscript, we are informed of the related work [36] before their publication, where they study the monopole harmonics on commutative sphere and fuzzy sphere.
} 


\section{Index theorem in spontaneously-symmetry- broken gauge theory on fuzzy 2-sphere}

\subsection{Review on fuzzy 2-sphere}

$\mathrm{NC}$ coordinates of the fuzzy 2-sphere are described by

$$
x_{i}=\alpha L_{i}
$$

where $\alpha$ is the NC parameter, and $L_{i}$ 's are $n$-dimensional irreducible representation matrices of $S U(2)$ algebra:

$$
\left[L_{i}, L_{j}\right]=i \epsilon_{i j k} L_{k}
$$

Then we have the following relation,

$$
\left(x_{i}\right)^{2}=\alpha^{2} \frac{n^{2}-1}{4} \mathbf{1}_{n} \equiv \rho^{2} \mathbf{1}_{n},
$$

where $\rho=\alpha \sqrt{\frac{n^{2}-1}{4}}$ expresses the radius of the fuzzy 2-sphere. The commutative limit can be taken by $\alpha \rightarrow 0, n \rightarrow \infty$ with $\rho$ fixed.

Any wave functions on the fuzzy 2 -sphere are mapped to $n \times n$ matrices. We can expand them in terms of NC analogues of the spherical harmonics $\hat{Y}_{l m}$, which are traceless symmetric products of the NC coordinates, and has an upper bound for the angular momentum $l$ as $l \leq n-1$. Derivatives along the Killing vectors of a function $M(\Omega)$ on the 2-sphere are written as the adjoint operator of $L_{i}$ on the corresponding matrix $\hat{M}$ :

$$
\mathcal{L}_{i} M(\Omega)=-i \epsilon_{i j k} x_{j} \partial_{k} M(\Omega) \leftrightarrow \tilde{L}_{i} \hat{M}=\left[L_{i}, \hat{M}\right]=\left(L_{i}^{L}-L_{i}^{R}\right) \hat{M}
$$

Here the superscript $\mathrm{L}(\mathrm{R})$ in $L_{i}$ means that this operator acts from the left (right) on the matrix $\hat{M}$. An integral of functions is given by a trace of matrices:

$$
\int \frac{d \Omega}{4 \pi} M(\Omega) \leftrightarrow \frac{1}{n} \operatorname{tr}[\hat{M}]
$$


Two types of Dirac operators, $D_{\mathrm{WW}}$ 37] and $D_{\mathrm{GKP}}[38$, 30], were constructed. $D_{\mathrm{Ww}}$ has doublers and the correct chiral anomaly cannot be reproduced. On the other hand, $D_{\mathrm{GKP}}$ breaks chiral symmetry at finite matrix size, and the chiral structures are not clear, though the chiral anomaly can be reproduced correctly in the commutative limit [39, 8, 40, 41]. We here review $D_{\mathrm{GKP}}$. The fermionic action is defined as

$$
\begin{aligned}
S_{\mathrm{GKP}} & =\operatorname{tr}\left[\bar{\Psi} D_{\mathrm{GKP}} \Psi\right], \\
D_{\mathrm{GKP}} & =\sigma_{i}\left(\tilde{L}_{i}+\rho a_{i}\right)+1,
\end{aligned}
$$

where $\sigma_{i}$ 's are Pauli matrices. The gauge field $a_{i}$ of $U(k)$ gauge group and the fermionic field $\Psi$ in the fundamental representation of the gauge group are expressed by $n k \times n k$ and $n k \times n$ matrices, respectively. This action is invariant under the gauge transformation:

$$
\begin{aligned}
\Psi & \rightarrow U \Psi \\
\bar{\Psi} & \rightarrow \bar{\Psi} U^{\dagger}, \\
a_{i} & \rightarrow U a_{i} U^{\dagger}+\frac{1}{\rho}\left(U L_{i} U^{\dagger}-L_{i}\right),
\end{aligned}
$$

since a combination, which sometimes called a covariant coordinate,

$$
A_{i} \equiv L_{i}+\rho a_{i}
$$

transforms covariantly as

$$
A_{i} \rightarrow U A_{i} U^{\dagger}
$$

In the commutative limit, the Dirac operator (2.7) becomes

$$
D_{\mathrm{GKP}} \rightarrow D_{\mathrm{com}}=\sigma_{i}\left(\mathcal{L}_{i}+\rho a_{i}\right)+1
$$

which is the ordinary Dirac operator on the commutative 2-sphere. The gauge fields $a_{i}$ 's in 3-dimensional space can be decomposed into the tangential compo- 
nents on the 2-sphere $a_{i}^{\prime}$ and the normal component $\phi$ as

$$
\begin{gathered}
\left\{\begin{array}{l}
a_{i}^{\prime}=\epsilon_{i j k} n_{j} a_{k}, \\
\phi=n_{i} a_{i},
\end{array}\right. \\
\Leftrightarrow a_{i}=-\epsilon_{i j k} n_{j} a_{k}^{\prime}+n_{i} \phi,
\end{gathered}
$$

where $n_{i}=x_{i} / \rho$ is a unit vector. The normal component $\phi$ is a scalar field on the 2-sphere. Then, the Dirac operator $D_{\text {com }}$ and $D_{\text {GKP }}$ have a coupling to the scalar field.

\subsection{GW Dirac operator and index theorem}

In order to discuss the chiral structures, a Dirac operator satisfying the GW relation is more suitable. Ref [18] provided a general prescription to define GW Dirac operator in arbitrary gauge field backgrounds on general finite NC geometries. We first define two chirality operators:

$$
\begin{aligned}
\Gamma^{R} & =a\left(\sigma_{i} L_{i}^{R}-\frac{1}{2}\right) \\
\hat{\Gamma} & =\frac{H}{\sqrt{H^{2}}}
\end{aligned}
$$

where

$$
H=a\left(\sigma_{i} A_{i}+\frac{1}{2}\right)
$$

$A_{i}$ is defined in (2.9), and

$$
a=\frac{2}{n}
$$

is introduced as a NC analogue of a lattice-spacing. These chirality operators satisfy

$$
\left(\Gamma^{R}\right)^{\dagger}=\Gamma^{R},(\hat{\Gamma})^{\dagger}=\hat{\Gamma},\left(\Gamma^{R}\right)^{2}=(\hat{\Gamma})^{2}=1
$$

In the commutative limit, both $\Gamma^{R}$ and $\hat{\Gamma}$ become the chirality operator on the commutative 2-sphere, $\gamma=n_{i} \sigma_{i}$.

We next define the GW Dirac operator as

$$
D_{\mathrm{GW}}=-a^{-1} \Gamma^{R}\left(1-\Gamma^{R} \hat{\Gamma}\right) .
$$


Then the action

$$
S_{\mathrm{GW}}=\operatorname{tr}\left[\bar{\Psi} D_{\mathrm{GW}} \Psi\right]
$$

is invariant under the gauge transformation (2.8). In the commutative limit, $D_{\mathrm{GW}}$ becomes

$$
D_{\mathrm{GW}} \rightarrow D_{\mathrm{com}}^{\prime}=\sigma_{i}\left(\mathcal{L}_{i}+\rho P_{i j} a_{j}\right)+1
$$

where $P_{i j}=\delta_{i j}-n_{i} n_{j}$ is the projector to the tangential directions on the sphere. Thus this Dirac operator $D_{\text {com }}^{\prime}$ is nothing but the Dirac operator on the commutative 2-sphere without coupling to the scalar field .

By the definition (2.19), the GW relation

$$
\Gamma^{R} D_{\mathrm{GW}}+D_{\mathrm{GW}} \hat{\Gamma}=0
$$

is satisfied. Then the following index theorem is satisfied:

$$
\operatorname{index}\left(D_{\mathrm{GW}}\right) \equiv\left(n_{+}-n_{-}\right)=\frac{1}{2} \mathcal{T} r\left(\Gamma^{R}+\hat{\Gamma}\right),
$$

where $n_{ \pm}$is the number of zero-modes of $D_{\mathrm{GW}}$ with positive or negative chirality

(for either $\Gamma^{R}$ or $\hat{\Gamma}$ ), and $\mathcal{T} r$ represents a trace over the space of matrices and over the spinor index. (See [18, 23] for a proof.)

The rhs of (2.23) has the following properties. Firstly, it takes only integer values since both $\Gamma^{R}$ and $\hat{\Gamma}$ have a form of sign operator by the definitions (2.14), (2.15). Secondly, it becomes the topological charge on the 2-sphere, the Chern character, in the commutative limit [18, 23]. Finally, it takes nonzero values for topologically nontrivial configurations if we slightly modify the definition of it, which we will see in the next subsections.

\subsection{Monopole configurations}

As topologically nontrivial configurations in the $U(2)$ gauge theory on the fuzzy 2-sphere, the following monopole configurations were constructed [22, 23]:

$$
A_{i}=\left(\begin{array}{cc}
L_{i}^{(n+m)} & \\
& L_{i}^{(n-m)}
\end{array}\right),
$$


where $A_{i}$ is defined in (2.9), and $L_{i}^{(n \pm m)}$ are $(n \pm m)$ dimensional irreducible representations of $S U(2)$ algebra 5 . The total matrix size is $N=2 n$. The $m=0$ case corresponds to two coincident fuzzy 2-spheres, whose effective action is given by the $U(2)$ gauge theory on the fuzzy 2 -sphere. The cases with general $m$ correspond to two fuzzy 2-spheres which share the same center but have the different radii. For $m \ll n$, as we will see below, they correspond to the monopole configurations with magnetic charge $-m$, where the gauge group $U(2)$ is spontaneously broken down to $U(1) \times U(1)$.

For the $m=1$ case, (2.24) is unitary and gauge equivalent to the following configuration:

$$
U A_{i} U^{\dagger}=L_{i}^{(n)} \otimes \mathbf{1}_{2}+\mathbf{1}_{n} \otimes \frac{\tau_{i}}{2},
$$

as is easily seen from the $S U(2)$ algebra. From (2.9), the first and the second terms represent the coordinate of the $\mathrm{NC}$ space and the configuration of the $U(2)$ gauge field respectively. Then, the gauge field is given by

$$
a_{i}=\frac{1}{\rho} \mathbf{1}_{n} \otimes \frac{\tau_{i}}{2}
$$

By taking the commutative limit of (2.26), and decomposing it into the normal and the tangential components of the sphere as in (2.12), it becomes

$$
\begin{aligned}
a_{i}^{\prime a} & =\frac{1}{\rho} \epsilon_{i j a} n_{j}, \\
\phi^{a} & =\frac{1}{\rho} n_{a},
\end{aligned}
$$

which is precisely the TP monopole configuration [23].

\subsection{Index theorem in the monopole backgrounds}

The index (2.23) turns out to vanish for the TP monopole configurations (2.24) with general $m$. The reason is as follows. TP monopole configuration breaks the

${ }^{5}$ Since (2.24) with $-m$ is unitary equivalent to the one with $m$, we will restrict $m \geq 0$ without loss of generality in this paper. 
$S U(2)$ gauge symmetry down to $U(1)$. Then the fermionic field in the fundamental representation which couples to the TP monopole background contains two components, corresponding to $+1 / 2$ and $-1 / 2$ electric charge of the unbroken $U(1)$ gauge group. Hence, these two components cancel the index. We thus need to pick up one of the two components in order to obtain nonzero index and chiral anomaly.

As in (2.23), the following equality is satisfied also in the projected spact 6 :

$$
\operatorname{index}\left(P^{(n \pm m)} D_{\mathrm{GW}}\right)=\frac{1}{2} \mathcal{T} r\left[P^{(n \pm m)}\left(\Gamma^{R}+\hat{\Gamma}\right)\right]
$$

where $P^{(n \pm m)}$ is the projection operator to pick up the Hilbert space for the $n \pm m$ dimensional representation in (2.24). That is, the projection operator picks up one of the two fuzzy 2 -spheres. The projection operator is written as

$$
\begin{aligned}
P^{(n \pm m)} & =\frac{\left(A_{i}\right)^{2}-\frac{1}{4}\left[(n \mp m)^{2}-1\right]}{\frac{1}{4}\left[(n \pm m)^{2}-1\right]-\frac{1}{4}\left[(n \mp m)^{2}-1\right]} \\
& =\frac{1}{2}(1 \pm T),
\end{aligned}
$$

where

$$
\begin{aligned}
T & =\frac{2}{n m}\left(A_{i}^{2}-\frac{n^{2}+m^{2}-1}{4}\right) \\
& =\left(\begin{array}{cc}
\mathbf{1}_{(n+m)} & \\
& -\mathbf{1}_{(n-m)}
\end{array}\right) .
\end{aligned}
$$

On the other hand, in the representation (2.9), (2.32) becomes

$$
T=\frac{2}{n m}\left(\rho\left\{L_{i}, a_{i}\right\}+\rho^{2} a_{i}^{2}-\frac{m^{2}}{4}\right) .
$$

In the commutative limit, it becomes $\frac{2 \rho}{m} \phi$ when $m \ll n$, where $\phi$ is the scalar field defined in (2.12). Then, $T$ is proportional to the scalar field. Also, $T$ is

${ }^{6}$ Note that the projection operator $P^{(n \pm m)}$ is written by the Casimir operator $\left(A_{i}\right)^{2}$ as in (2.30). Then, we can see from the definition (2.14) and (2.15) that $\left[P^{(n \pm m)}, \Gamma^{R}\right]=\left[P^{(n \pm m)}, \hat{\Gamma}\right]=$ 0 . Also, from (2.19), we can see $\left[P^{(n \pm m)}, D_{\mathrm{GW}}\right]=0$. 
normalized as $T^{2}=\mathbf{1}_{2 n}$, which can be seen from (2.33). Therefore, $T$ is the generator for the unbroken $U(1)$ gauge group in the TP monopole. Then, the eigenstate of $T$ with eigenvalue \pm 1 corresponds to the fermionic state with $\pm 1 / 2$ electric charge of the unbroken $U(1)$ gauge group 7 . Thus the projection operator $P^{(n \pm m)}$ picks up $\pm 1 / 2$ electric charge component.

Then, the index in the projected space (2.29) corresponds to the index for each electric charge component, which is precisely what we needed to define as we mentioned at the beginning of this subsection. For the configurations (2.24), we can see [22, 23]

$$
\frac{1}{2} \mathcal{T} r\left[P^{(n \pm m)}\left(\Gamma^{R}+\hat{\Gamma}\right)\right]=\mp m .
$$

We thus obtain nonzero index $\mp m$ for $\pm 1 / 2$ electric charge component. Without the projection operator, contributions from $+1 / 2$ and $-1 / 2$ charges cancel the index.

In the commutative limit,

$$
\begin{aligned}
\frac{1}{2} \mathcal{T} r\left[P^{(n \pm m)}\left(\Gamma^{R}+\hat{\Gamma}\right)\right] & =\frac{1}{2} \mathcal{T} r\left[\frac{1}{2}(1 \pm T)\left(\Gamma^{R}+\hat{\Gamma}\right)\right] \\
& = \pm \frac{1}{2} \mathcal{T} r\left[\frac{1}{2} T\left(\Gamma^{R}+\hat{\Gamma}\right)\right] \\
& \longrightarrow \pm \frac{\rho^{2}}{8 \pi} \int_{S^{2}} d \Omega \epsilon_{i j k} n_{i} \phi^{\prime a} F_{j k}^{a}
\end{aligned}
$$

where $\phi^{\prime a}$ is a scalar field normalized as $\sum_{a}\left(\phi^{\prime a}\right)^{2}=1 . F_{j k}=F_{j k}^{a} \tau^{a} / 2$ is the field strength defined as $F_{j k}=\partial_{j} a_{k}^{\prime}-\partial_{k} a_{j}^{\prime}-i\left[a_{j}^{\prime}, a_{k}^{\prime}\right]$. This is the magnetic charge for the unbroken $U(1)$ component in the TP monopole configuration, which is nothing but the topological charge for the TP monopole configuration 8 . Compared with (2.35), the topological charge defined by inserting $\phi^{\prime}$ as in (2.36) turns out to be $-|m|$ for the configurations (2.24), as in [28].

${ }^{7}$ Strictly speaking, since $T \sim \frac{2 \rho}{m} \phi$, the eigenstate of $T$ with eigenvalue \pm 1 corresponds to electric charge $\pm 1 / 2(\mp 1 / 2)$ for $m>0(m<0)$. Anyway, we restrict $m \geq 0$ in this paper.

8 The topological charge should have an additional term as the second term in (2.50). However the additional term vanishes for the TP monopole configurations. 
Finally we see the gauge symmetry breaking in the configurations (2.24) with $m \geq 1: U(2) \rightarrow U(1) \times U(1)$. There are two ways to look at the unbroken gauge symmetries:

1. Each sphere in (2.24) has unbroken $U(1)$ symmetry, and totally (2.24) has $U(1) \times U(1)$ symmetry. The generator for each $U(1)$ is written as

$$
\left(\begin{array}{ll}
\mathbf{1}_{(n+m)} & \\
& 0
\end{array}\right),\left(\begin{array}{ll}
0 & \\
& \mathbf{1}_{(n-m)}
\end{array}\right) .
$$

2. $U(2) \simeq S U(2) \times U(1)$, and the $S U(2)$ breaks down to $U(1)$ in the TP monopole configuration. We can rearrange the generators (2.37) as

$$
\mathbf{1}_{2 n}, T,
$$

where $T$ is given by (2.33). On the other hand, in the representation (2.34), $T$ is identified as noncommutative generalization of the unbroken $U(1)$ generator for the commutative TP monopole configurations.

The above two ways of looking at the unbroken gauge symmetry are equivalent since the representations (2.24) and (2.9) are unitary and gauge equivalent.

\subsection{Extension to general configurations}

In the previous subsection, we have considered the index theorem (2.29) for the monopole background configurations (2.24), which satisfy the equation of motion. We now extend it to general configurations which do not necessarily satisfy the equation of motion. The only assumption in the following is that the $U(2)$ gauge symmetry is spontaneously broken to $U(1) \times U(1)$ through the Higgs mechanism, i.e. a nonzero value of the scalar field. Under this assumption, the gauge configuration space on the fuzzy 2-sphere can be classified into the topological sectors.

\footnotetext{
${ }^{9}$ We gave the similar argument in [28] for $m=1$ case. We here generalize it to $m \geq 1$.
} 
We first generalize the definition of the electric charge operator $T$ to

$$
T^{\prime}=\frac{\left(A_{i}\right)^{2}-\frac{n^{2}-1}{4}}{\sqrt{\left[\left(A_{i}\right)^{2}-\frac{n^{2}-1}{4}\right]^{2}}} .
$$

This definition is valid for general configurations $A_{i}$ unless the denominator has zero-modes. It satisfies

$$
\left(T^{\prime}\right)^{\dagger}=T^{\prime},\left(T^{\prime}\right)^{2}=1,
$$

and its eigenvalue takes 1 or -1 . The commutative limit of $T^{\prime}$ becomes the normalized scalar field as

$$
T^{\prime} \rightarrow 2 \phi^{\prime}=2 \phi^{\prime a} \frac{\tau^{a}}{2} .
$$

For the configurations (2.24), $T^{\prime}$ reduces to the previous one (2.33).

We next define modified chirality operators as

$$
\begin{aligned}
\Gamma^{\prime} & =\frac{\left\{T^{\prime}, \Gamma^{R}\right\}}{2}=T^{\prime} \Gamma^{R}, \\
\hat{\Gamma}^{\prime} & =\frac{\left\{T^{\prime}, \hat{\Gamma}\right\}}{\sqrt{\left\{T^{\prime}, \hat{\Gamma}\right\}^{2}}} .
\end{aligned}
$$

These chirality operators are weighted by the electric charge operator $T^{\prime}$ but they still satisfy the usual relations:

$$
\left(\Gamma^{\prime}\right)^{\dagger}=\Gamma^{\prime},\left(\hat{\Gamma}^{\prime}\right)^{\dagger}=\hat{\Gamma}^{\prime},\left(\Gamma^{\prime}\right)^{2}=\left(\hat{\Gamma}^{\prime}\right)^{2}=1 .
$$

We then define a modified GW Dirac operator as

$$
D_{\mathrm{GW}}^{\prime}=-a^{-1} \Gamma^{\prime}\left(1-\Gamma^{\prime} \hat{\Gamma}^{\prime}\right) .
$$

This Dirac operator is also weighted by the electric charge operator $T^{\prime}$, which avoids the cancellation between the contributions from $\pm 1 / 2$ electric charge components when we consider its index. In the commutative limit, we obtain

$$
D_{\mathrm{GW}}^{\prime} \rightarrow \frac{1}{2}\left\{2 \phi^{\prime}, D_{\mathrm{com}}^{\prime}\right\} .
$$


In the $\phi^{\prime a}=(0,0,1)$ gauge, it becomes

$$
\tau^{3}\left(\sigma_{i} \mathcal{L}_{i}+1+\rho \sigma_{i} P_{i j} a_{j}^{3} \frac{\tau^{3}}{2}\right)
$$

Inside of the parenthesis is precisely the Dirac operator with coupling to the unbroken $U(1)$ gauge field.

From the definition (2.45), this Dirac operator satisfies the GW relation

$$
\Gamma^{\prime} D_{\mathrm{GW}}^{\prime}+D_{\mathrm{GW}}^{\prime} \hat{\Gamma}^{\prime}=0
$$

and thus the index theorem

$$
\frac{1}{2} \operatorname{index}\left(D_{\mathrm{GW}}^{\prime}\right)=\frac{1}{4} \mathcal{T} r\left[\Gamma^{\prime}+\hat{\Gamma}^{\prime}\right]
$$

can be proved similarly to the ordinary case. In the commutative limit, the rhs turns out to become

$$
\frac{1}{4} \mathcal{T} r\left[\Gamma^{\prime}+\hat{\Gamma}^{\prime}\right] \rightarrow \frac{\rho^{2}}{8 \pi} \int_{S^{2}} d \Omega \epsilon_{i j k} n_{i}\left(\phi^{\prime a} F_{j k}^{a}-\epsilon_{a b c} \phi^{\prime a}\left(D_{j} \phi^{\prime b}\right)\left(D_{k} \phi^{\prime c}\right)\right)
$$

which is precisely the topological charge for the configurations with unbroken $U(1)$ gauge symmetry [42].

For the configurations (2.24),$T^{\prime}$ commutes with with $\hat{\Gamma}$. Then we obtain $\hat{\Gamma}^{\prime}=T^{\prime} \hat{\Gamma}$. Then the above index reduces to the previous one;

$$
\frac{1}{4} \mathcal{T} r\left[\Gamma^{\prime}+\hat{\Gamma}^{\prime}\right]=\frac{1}{2} \mathcal{T} r\left[\frac{1}{2} T\left(\Gamma^{R}+\hat{\Gamma}\right)\right]
$$

Hence the index theorem (2.49) gives a natural generalization for general configurations which are not restricted to the special solutions such as the TP monopoles.

Finally we consider a condition for gauge configurations that the topological charge (2.49) can be well-defined.

In the commutative limit, we obtain

$$
\left(A_{i}\right)^{2}-\frac{n^{2}-1}{4} \rightarrow \rho n \phi(x)=\rho n \phi(x)^{a} \frac{\tau^{a}}{2} .
$$


Then,

$$
\left[\left(A_{i}\right)^{2}-\frac{n^{2}-1}{4}\right]^{2} \rightarrow \frac{(\rho n)^{2}}{4}\left[\sum_{a}\left(\phi^{a}(x)\right)^{2} \mathbf{1}_{2}+\mathcal{O}(1 / n)\right] .
$$

In order to define the topological sectors by using the unbroken $U(1)$ gauge symmetry in the commutative theory, the scalar field should take non-vanishing values on arbitrary points on the sphere, namely, $\rho^{2} \sum_{a}\left(\phi^{a}(x)\right)^{2} \sim \mathcal{O}(1)$ for all $x$. Otherwise we could not define the unbroken $U(1)$ direction, nor could we define the topological charge (2.50). This condition corresponds to

$$
\left[\left(A_{i}\right)^{2}-\frac{n^{2}-1}{4}\right]^{2} \sim \mathcal{O}\left(n^{2}\right)
$$

which means that all of the eigenvalues are of the order of $n^{2}$. Smaller eigenvalues may invalidate the definition of topology, while larger eigenvalues may change the structure of space and violate the assumption that we define the gauge theory on the fuzzy 2-sphere. The upper bound on the eigenvalues corresponds to the admissibility condition, which was introduced to assure the topological structure in the lattice gauge theory [43, 44, 45]. The condition (2.54) has also the lower bound, then it gives an extension of the admissibility condition.

More detailed analysis of this subsection will be reported in a separated paper [46].

\section{Spectrum of the GW Dirac operator}

In this section, we will calculate the spectrum for the Ginsparg-Wilson Dirac operator (2.19) in the monopole backgrounds (2.24):

$$
D_{\mathrm{GW}}^{m}=\left(\begin{array}{ll}
\frac{n}{n+m}\left(\sigma_{i} L_{i}^{(n+m)}+\frac{1}{2}\right) & \\
& \frac{n}{n-m}\left(\sigma_{i} L_{i}^{(n-m)}+\frac{1}{2}\right)
\end{array}\right)-\left(\sigma_{i} L_{i}^{R}-\frac{1}{2}\right) .
$$


We define the total angular momentum operator

$$
\begin{aligned}
M_{i} & =L_{i}+a_{i}-L_{i}^{R}+\frac{\sigma_{i}}{2} \\
& =A_{i}-L_{i}^{R}+\frac{\sigma_{i}}{2} \\
& =\left(\begin{array}{c}
L_{i}^{(n+m)} \\
L_{i}^{(n-m)}
\end{array}\right)-L_{i}^{R}+\frac{\sigma_{i}}{2} .
\end{aligned}
$$

We also consider the electric charge operator $T$ defined in (2.32), and the chirality operator $\Gamma^{R}$ defined in (2.14). Since $M_{i}, T, \Gamma^{R}$ commute with one another, we can consider the simultaneous eigenstates for these operators a: 10

$$
\begin{aligned}
M_{i}^{2}\left|J, J_{3}, \delta, \nu\right\rangle & =J(J+1)\left|J, J_{3}, \delta, \nu\right\rangle, \\
M_{3}\left|J, J_{3}, \delta, \nu\right\rangle & =J_{3}\left|J, J_{3}, \delta, \nu\right\rangle, \\
T\left|J, J_{3}, \delta, \nu\right\rangle & =\delta\left|J, J_{3}, \delta, \nu\right\rangle, \\
\Gamma^{R}\left|J, J_{3}, \delta, \nu\right\rangle & =\nu\left|J, J_{3}, \delta, \nu\right\rangle .
\end{aligned}
$$

We here note that the state with $\delta= \pm 1$ is the state with spin $L \pm m / 2$ for the operator $A_{i}$, where $L$ is taken to be $\frac{n-1}{2}$. The state with $\nu= \pm 1$ is the state with spin $L \mp 1 / 2$ for the operator $-L_{i}^{R}+\frac{\sigma_{i}}{2}$. Thus, from (3.3), $J$ takes the values given in Table 1, Here we assumed $m>0$. Since the total number of these eigenstates is

$$
\begin{aligned}
& 2\left[2 \cdot \frac{m-1}{2}+1\right]+4\left[\sum_{J=\frac{m+1}{2}}^{2 L-\frac{m+1}{2}}(2 J+1)\right]+3\left[2\left(2 L-\frac{m-1}{2}\right)+1\right] \\
& +2\left[\sum_{J=2 L-\frac{m}{2}+\frac{3}{2}}^{2 L+\frac{m-1}{2}}(2 J+1)\right]+\left[2\left(2 L+\frac{m+1}{2}\right)+1\right] \\
& =4 n^{2},
\end{aligned}
$$

10 Since $M_{i}, T$, and $\hat{\Gamma}$ also commute with one another, we can consider the simultaneous eigenstates for them, and follow the same calculations that will be performed in this paper by using $M_{i}, T$, and $\Gamma^{R}$. 
Table 1: Values of $J$ in each $\delta, \nu$ sector. We assume $m>0$ here.

\begin{tabular}{|c|ccccc|}
\hline & $\delta(T)$ & - & - & + & + \\
$J$ & $\nu\left(\Gamma^{R}\right)$ & + & - & + & - \\
\hline$\frac{m-1}{2}$ & & $\circ$ & & & $\circ$ \\
$\frac{m+1}{2}$ & & $\circ$ & $\circ$ & $\circ$ & $\circ$ \\
$\frac{m+3}{2}$ & & $\circ$ & $\circ$ & $\circ$ & $\circ$ \\
$\vdots$ & $\vdots$ & $\vdots$ & $\vdots$ & $\vdots$ \\
$2 L-\frac{m+1}{2}$ & & $\circ$ & 0 & $\circ$ & $\circ$ \\
$2 L-\frac{m-1}{2}$ & & & 0 & $\circ$ & $\circ$ \\
$\vdots$ & & & & $\vdots$ & $\vdots$ \\
$2 L+\frac{m-1}{2}$ & & & & & \\
$2 L+\frac{m+1}{2}$ & & & & 0 & $\circ$ \\
\hline
\end{tabular}

these eigenstates exhaust the complete set of the Hilbert space. Note that the spectrum starts from the nonzero lowest spin, $J=\frac{m-1}{2}$, as in the case of the monopole harmonics. Note also that, for the lowest spin states with $J=\frac{m-1}{2}$, and for the highest spin states with $(J, \delta)=\left(2 L-\frac{m-1}{2},-1\right),\left(2 L+\frac{m+1}{2},+1\right)$, only $\nu=+1$ or $\nu=-1$ exists for each $\delta$, while for the other states, both $\nu=+1$ and $\nu=-1$ exist for each 1112 .

\footnotetext{
11 The unbalance between $\nu=+1$ and $\nu=-1$ in the total spectrum is consistent with $\mathcal{T} r\left(\Gamma^{R}\right)=-4 n$. The unbalance between $\delta=+1$ and $\delta=-1$ in the total spectrum is consistent with $\mathcal{T} r(T)=4 n m$.

${ }^{12}$ We can also consider the simultaneous eigenstates for $M_{i}, T$, and $\hat{\Gamma}$, and make the same table as Table 1. For the lowest spin states, which is shown to be zero-modes of $D_{\mathrm{GW}}, \pm$ eigenvalue for $\Gamma^{R}$ corresponds to \pm eigenvalue for $\hat{\Gamma}$, as can be seen from the definition of $D_{\mathrm{GW}}$ (2.19). For the highest spin states, \pm eigenvalue for $\Gamma^{R}$ corresponds to $\mp$ eigenvalue for $\hat{\Gamma}$, as can be seen from the GW relation (2.22).
} 
By straightforward calculations, square of $D_{\mathrm{GW}}^{m}$ becomes

$$
\left(D_{\mathrm{GW}}^{m}\right)^{2}=\frac{n}{n+m T}\left[M_{i}^{2}-\frac{m^{2}-1}{4}\right]
$$

and $\left(D_{\mathrm{GW}}^{m}\right)^{2}$ commutes with $M_{i}, T, \Gamma^{R}$. We thus obtain the spectrum for $\left(D_{\mathrm{GW}}^{m}\right)^{2}$ as follows:

$$
\left(D_{\mathrm{GW}}^{m}\right)^{2}\left|J, J_{3}, \delta, \nu\right\rangle=\frac{n}{n+m \delta}\left[J(J+1)-\frac{m^{2}-1}{4}\right]\left|J, J_{3}, \delta, \nu\right\rangle .
$$

Note that the states with the lowest spin $J=\frac{m-1}{2}$ correspond to the zero-modes for the $D_{\mathrm{GW}}^{m}$.

We can also show

$$
\begin{aligned}
{\left[M_{i}, D_{\mathrm{GW}}^{m}\right] } & =0, \\
{\left[T, D_{\mathrm{GW}}^{m}\right] } & =0, \\
{\left[\Gamma^{R}, D_{\mathrm{GW}}^{m}\right] } & \neq 0 .
\end{aligned}
$$

Therefore, linear combinations over $\nu$ for each $J, J_{3}, \delta, \sum_{\nu} c_{\nu}\left|J, J_{3}, \delta, \nu\right\rangle$, give eigenstates for the Dirac operator $D_{\mathrm{GW}}^{m}$ as

$$
D_{\mathrm{GW}}^{m}\left[\sum_{\nu} c_{\nu}\left|J, J_{3}, \delta, \nu\right\rangle\right]= \pm \sqrt{\frac{n}{n+m \delta}\left[J(J+1)-\frac{m^{2}-1}{4}\right]}\left[\sum_{\nu} c_{\nu}\left|J, J_{3}, \delta, \nu\right\rangle\right]
$$

By the Ginsparg-Wilson relation,

$$
\begin{aligned}
& \Gamma^{R} D_{\mathrm{GW}}^{m}+D_{\mathrm{GW}}^{m} \hat{\Gamma}=0, \\
& D_{\mathrm{GW}}^{m} \Gamma^{R}+\hat{\Gamma} D_{\mathrm{GW}}^{m}=0,
\end{aligned}
$$

if $\sum_{\nu} c_{\nu}\left|J, J_{3}, \delta, \nu\right\rangle$ is an eigenstate for the $D_{\mathrm{GW}}^{m}$ with eigenvalue $\alpha$, $\left(\Gamma^{R}+\hat{\Gamma}\right) \sum_{\nu} c_{\nu}\left|J, J_{3}, \delta, \nu\right\rangle$ is an eigenstate with eigenvalue $-\alpha$. From (2.19), we see $\Gamma^{R}+\hat{\Gamma}=a D_{\mathrm{GW}}^{m}+2 \Gamma^{R}$. Then,

$$
\left(\Gamma^{R}+\hat{\Gamma}\right) \sum_{\nu} c_{\nu}\left|J, J_{3}, \delta, \nu\right\rangle=(a \alpha+2) c_{1}\left|J, J_{3}, \delta, 1\right\rangle+(a \alpha-2) c_{-1}\left|J, J_{3}, \delta,-1\right\rangle
$$


For the states with the highest spin, $\left(J=2 L-\frac{m-1}{2}, \delta=-1\right)$ and $\left(J=2 L+\frac{m+1}{2}\right.$, $\delta=1)$ in Table 1, only $\nu=-1$ exists. Hence $\left(\Gamma^{R}+\hat{\Gamma}\right)\left|J, J_{3}, \delta, \nu=-1\right\rangle$ must vanish. From (3.18) we obtain $a \alpha=2$. Thus only positive eigenvalues for $D_{\mathrm{GW}}^{m}$ exist for the highest spin states. For the other states, since $\alpha=$ $\pm \sqrt{\frac{n}{n+m \delta}\left[J(J+1)-\frac{m^{2}-1}{4}\right]}$, we see $-2<a \alpha<2$. Since $\left(\Gamma^{R}+\hat{\Gamma}\right) \sum_{\nu} c_{\nu}\left|J, J_{3}, \delta, \nu\right\rangle$ does not vanish, both positive and negative eigenvalues exist. We illustrate the spectrum for the case with $n=10$ and $m=0,1,2,3,4$ in Figure 1,

Next, we determine the coefficients $c_{\nu}$, and obtain the form of the eigenstates for the nonzero eigenvalues of $D_{\mathrm{GW}}^{m}$. Since $\left(\Gamma^{R}+\hat{\Gamma}\right) \sum_{\nu} c_{\nu}\left|J, J_{3}, \delta, \nu\right\rangle$ must be orthogonal to $\sum_{\nu} c_{\nu}\left|J, J_{3}, \delta, \nu\right\rangle$,

$$
(a \alpha+2)\left|c_{1}\right|^{2}+(a \alpha-2)\left|c_{-1}\right|^{2}=0 .
$$

Therefore, the eigenstates for the eigenvalue $\alpha$ are

$$
\frac{1}{2}\left[\sqrt{2-a \alpha}\left|J, J_{3}, \delta, 1\right\rangle+\frac{\alpha}{|\alpha|} \sqrt{2+a \alpha}\left|J, J_{3}, \delta,-1\right\rangle\right]
$$

where $\alpha= \pm \sqrt{\frac{n}{n+m \delta}\left[J(J+1)-\frac{m^{2}-1}{4}\right]}$. Here we have absorbed the relative phase into the definition of $\left|J, J_{3}, \delta, 1\right\rangle$ and $\left|J, J_{3}, \delta,-1\right\rangle$.

Next, we check the index theorem (2.29) by counting the number of the chiral zero-modes of $D_{\mathrm{GW}}^{m}$. As noted before, the zero-modes for $D_{\mathrm{GW}}^{m}$ correspond to the states with $J=\frac{m-1}{2}$, whose degeneracy is $m$. Then, for $(\delta, \nu)=(+1,-1)$ in Table 1, the index in the projected space is given by

$$
\operatorname{Index}\left(P^{(n+m)} D_{\mathrm{GW}}\right)=n_{+}-n_{-}=0-m=-m .
$$

For $(\delta, \nu)=(-1,+1)$, it is given by

$$
\operatorname{Index}\left(P^{(n-m)} D_{\mathrm{GW}}\right)=n_{+}-n_{-}=m-0=m .
$$

Comparing with (2.35), this is consistent with the index theorem (2.29).

Finally, we give some comments about the highest spin states. As we noted above, the highest spin sates give only positive eigenvalue of $D_{\mathrm{GW}}^{m}$, and thus the 


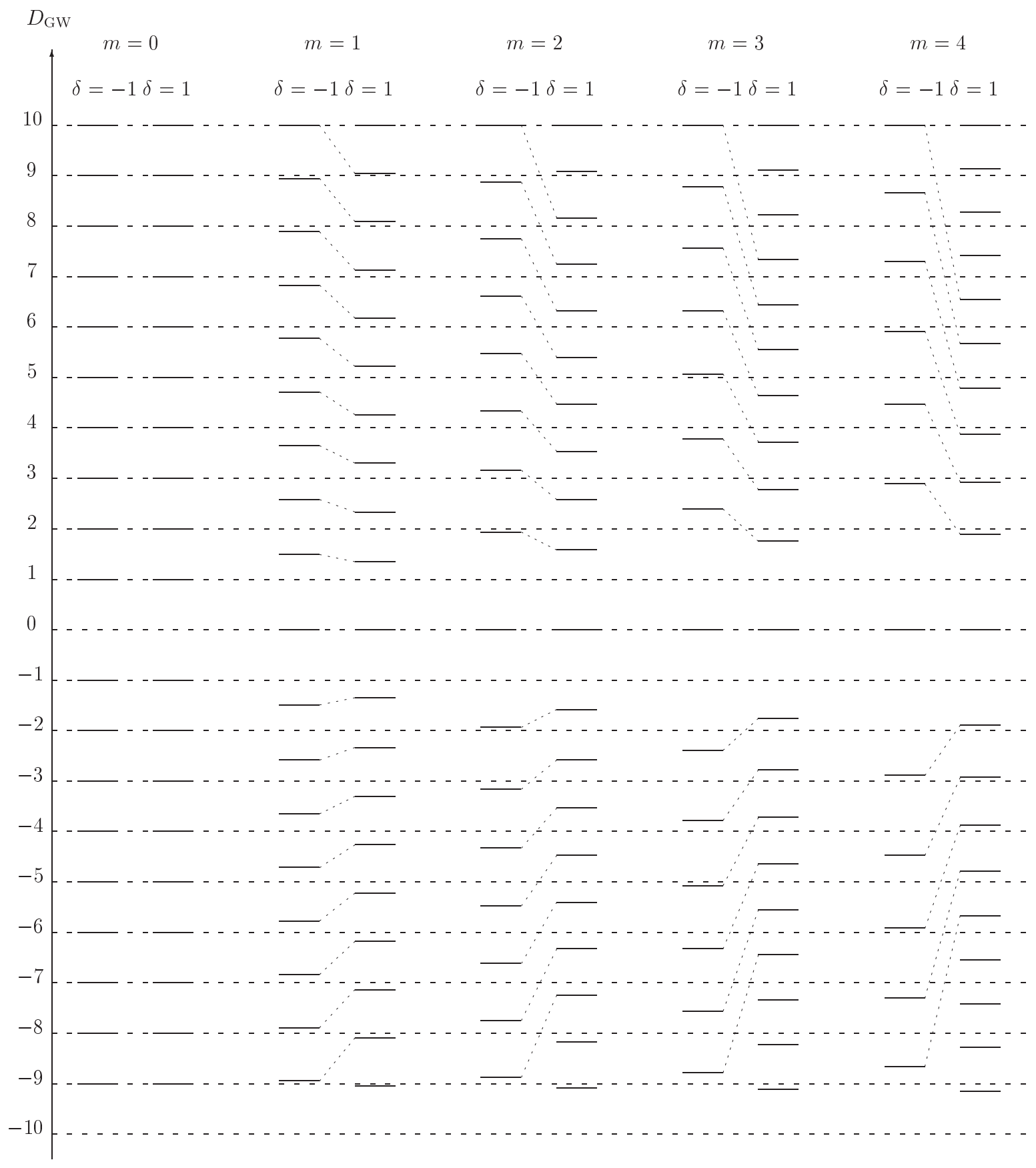

Figure 1: Spectrum for GW Dirac operator for the cases of $n=10$ and $m=$ $0,1,2,3,4$. Doted line connects the states with the same value of $J$. 
largest eigenvalue of the GW Dirac operator takes only a positive value (see the spectrum in Figure 1). This unbalance between the positive and the negative eigenvalues of $D_{\mathrm{GW}}$ comes from the fact that $D_{\mathrm{GW}}$ does not satisfy the ordinary chiral symmetry, i.e. $\left\{D_{\mathrm{GW}}, \Gamma^{R}\right\} \neq 0$. It is known that the highest spin states correspond to the species doublers of the Watamura's Dirac operator $D_{\mathrm{Ww}}$ [37].

As can be seen from Table 1, these modes have only $\nu=-1$, which means that they have definite chirality defined by $\Gamma^{R}$. The similar pattern of spectrum was provided for the GW Dirac operator $D$ in the lattice gauge theories [47]:

i) $n_{ \pm}$states with eigenvalue zero for $\gamma_{5} D$ and \pm 1 for the chirality $\gamma_{5}$.

ii) $N_{ \pm}$states with eigenvalue $\pm \frac{2}{a}$ for $\gamma_{5} D$ and \pm 1 for the chirality $\gamma_{5}$.

iii) The remaining states have eigenvalues $\pm \alpha_{n}$ for $\gamma_{5} D$ pairwise, with $0<\left|\alpha_{n}\right|<$ $\frac{2}{a}$.

It was also shown that the $N_{ \pm}$would-be species doubler sates play an important role in defining the index consistently. As the index theorem states, in the well-defined continuum theories, the trace of the chirality operator becomes $\mathcal{T} r_{\text {con }}\left(\gamma_{5}\right)=n_{+}-n_{-}$, while in the lattice theories $\mathcal{T} r\left(\gamma_{5}\right)=0$. This discrepancy can be solved by taking account of the contributions from the $N_{ \pm}$would-be species doublers. Then $\mathcal{T} r\left(\gamma_{5}\right)=n_{+}-n_{-}+N_{+}-N_{-}=0$ is actually satisfied on the lattice. The would-be species doublers can be eliminated by adopting $\frac{1}{2} \mathcal{T} r\left(\gamma_{5}+\hat{\gamma}_{5}\right)=\mathcal{T} r\left(\gamma_{5}\left(1-\frac{1}{2} a D_{\mathrm{GW}}\right)\right)$ instead of $\mathcal{T} r\left(\gamma_{5}\right)$, or by inserting some other factor which suppresses the contributions from the large eigenvalues of the Dirac operator. Then the correct value of the index is obtained, and the smooth continuum limit can be taken. Actually, these features of the spectrum and the index are given only by the GW algebra, and they hold in the present case of the fuzzy 2-sphere as well.

Now let us come back to the case of the fuzzy 2-sphere, and consider the counterpart of $\mathcal{T} r\left(\gamma_{5}\right)$, which is $\mathcal{T} r\left(\Gamma^{R}\right)$. From Table 1, for the $\delta=1$ sector, we can see that $n_{+}-n_{-}=-m, N_{+}-N_{-}=-(2 n+m)$. For the other states, both 
values of $\nu$ exist pairwise. Thus

$$
\mathcal{T} r\left(P^{(n+m)} \Gamma^{R}\right)=\left(n_{+}-n_{-}\right)+\left(N_{+}-N_{-}\right)=-m-(2 n+m)=-2(n+m) .
$$

In the same way, for the $\delta=-1$ sector, we see

$$
\mathcal{T} r\left(P^{(n-m)} \Gamma^{R}\right)=\left(n_{+}-n_{-}\right)+\left(N_{+}-N_{-}\right)=m-(2 n-m)=-2(n-m) .
$$

We can eliminate the contributions from the would-be species doublers by adopt$\operatorname{ing} \frac{1}{2} \mathcal{T} r\left(P^{(n \pm m)}\left(\Gamma^{R}+\hat{\Gamma}\right)\right)=\mathcal{T} r\left(P^{(n \pm m)}\left(\Gamma^{R}+\frac{1}{2} a D_{\mathrm{GW}}\right)\right)$, as in the lattice gauge theories. However, (3.23) and (3.24) do not vanish, while $\mathcal{T} r\left(\gamma_{5}\right)=0$ in the lattice gauge theories. This is because the definition of $\Gamma^{R}$ in (2.14) has a constant term $-\frac{a}{2}=-\frac{1}{n}$, and thus $\mathcal{T} r\left(P^{(n \pm m)} \Gamma^{R}\right)$ has non-vanishing value, which is $-\frac{1}{n}$ times the dimension of the Hilbert space, $2 n(n \pm m)$. These non-vanishing values reflect the noncommutativity of the geometry which can be interpreted as introducing a magnetic flux.

\section{Chiral zero-modes}

In this section we will obtain the form of the chiral zero-modes for the GW Dirac operator in the TP monopole background with $m=1$. By the unitary transformation (2.25), (3.1) becomes

$$
\begin{aligned}
D_{\mathrm{GW}}^{\mathrm{TP}} & \equiv U D_{\mathrm{GW}}^{m=1} U^{\dagger} \\
& =\sigma_{i} \tilde{L}_{i}+1+\sigma_{i} \frac{\tau_{i}}{2}-\frac{1}{n^{2}-1} L_{i} \tau_{i}\left[1+2 \sigma_{j}\left(L_{j}+\frac{\tau_{j}}{2}\right)\right]
\end{aligned}
$$

The benefit of taking this representation is that we can easily see the correspondence between the noncommutative and commutative theories.

As we showed in section 3 , the zero-modes for $D_{\mathrm{GW}}^{\mathrm{TP}}$ correspond to the lowest spin states with $J=\frac{m-1}{2}$, which is $J=0$ for $m=1$. They thus can be written as $\epsilon_{\alpha l}$ and $L^{i} \sigma_{\alpha \alpha^{\prime}}^{i} \epsilon_{\alpha^{\prime} l}$, where $\alpha$ and $l$ are spinor and gauge group indices respectively. 
Indeed, we can show directly that these modes are zero-modes of $D_{\mathrm{GW}}^{\mathrm{TP}}$ by using the identity:

$$
\sigma^{i} \epsilon=-\epsilon\left(\tau^{i}\right)^{T}
$$

or, if the indices written explicitly,

$$
\sigma_{\alpha \alpha^{\prime}}^{i} \epsilon_{\alpha^{\prime} l}=-\tau_{l l^{\prime}}^{i} \epsilon_{\alpha l^{\prime}}
$$

For example,

$$
\begin{aligned}
\left(D_{\mathrm{GW}}^{\mathrm{TP}} \epsilon\right)_{\alpha l} & =\epsilon_{\alpha l}+\frac{1}{2} \sigma_{\alpha \alpha^{\prime}}^{i} \tau_{l l^{\prime}}^{i} \epsilon_{\alpha^{\prime} l^{\prime}}-\frac{1}{n^{2}-1}\left[L^{i} \tau_{l l^{\prime}}^{i} \epsilon_{\alpha l^{\prime}}+2 L^{i} L^{j} \sigma_{\alpha \alpha^{\prime}}^{j} \tau_{l l^{\prime}}^{i} \epsilon_{\alpha^{\prime} l^{\prime}}+L^{i} \sigma_{\alpha \alpha^{\prime}}^{j} \tau_{l l^{\prime}}^{i} \tau_{l^{\prime} l^{\prime}}^{j} \epsilon_{\alpha^{\prime} l^{\prime \prime}}\right] \\
& =\epsilon_{\alpha l}-\frac{1}{2}\left(\sigma^{i} \sigma^{i} \epsilon\right)_{\alpha l}-\frac{1}{n^{2}-1}\left[-L^{i}\left(\sigma^{i} \epsilon\right)_{\alpha l}-2 L^{i} L^{j}\left(\sigma^{j} \sigma^{i} \epsilon\right)_{\alpha l}+L^{i}\left(\sigma^{j} \sigma^{j} \sigma^{i} \epsilon\right)_{\alpha l}\right] \\
& =\epsilon_{\alpha l}-\frac{3}{2} \epsilon_{\alpha l}-\frac{1}{n^{2}-1}\left[2 L^{i}\left(\sigma^{i} \epsilon\right)_{\alpha l}-\frac{n^{2}-1}{2} \epsilon_{\alpha l}-2 L^{i}\left(\sigma^{i} \epsilon\right)_{\alpha l}\right] \\
& =0 .
\end{aligned}
$$

In the same way, we can show $\left(D_{\mathrm{GW}}^{\mathrm{TP}} L^{i} \sigma^{i} \epsilon\right)_{\alpha l}=0$.

Chiral zero-modes can be obtained by the linear combinations of these two zero-modes $\epsilon_{\alpha l}$ and $L^{i} \sigma_{\alpha \alpha^{\prime}}^{i} \epsilon_{\alpha^{\prime} l}$. The states with $(\delta, \nu)=(\mp 1, \pm 1)$ are given by

$$
\mid J=0, \delta=\mp 1, \nu= \pm 1>=\frac{1}{2}\left(1 \pm \Gamma^{R}\right) \epsilon=\frac{1}{2}(1 \mp T) \epsilon
$$

or, if the indices written explicitly,

$\mid J=0, \delta=\mp 1, \nu= \pm 1>=\frac{1}{2}\left[\left(1 \mp \frac{1}{n}\right) \epsilon_{\alpha l} \pm \frac{2}{n} L^{i} \sigma_{\alpha \alpha^{\prime}}^{i} \epsilon_{\alpha^{\prime} l}\right]=\frac{1}{2}\left[\left(1 \mp \frac{1}{n}\right) \epsilon_{\alpha l} \mp \frac{2}{n} L^{i} \tau_{l l^{\prime}}^{i} \epsilon_{\alpha l^{\prime}}\right]$,

where again we used the identity (4.3).

In appendix B, we calculate the spectrum of the Dirac operator in the commutative theory. We also obtain the chiral zero-modes in (B.19):

$$
\mid J=0, \delta=\mp 1, \nu= \pm 1>_{\mathrm{com}}=\frac{1}{2}\left[1 \pm n^{i} \sigma^{i}\right]_{\alpha \alpha^{\prime}} \epsilon_{\alpha^{\prime} l}=\frac{1}{2}\left[1 \mp n^{i} \tau^{i}\right]_{l l^{\prime}} \epsilon_{\alpha l^{\prime}}
$$

where $n^{i}=x^{i} / \rho$ is a unit vector. As we mentioned before, the correspondence between the noncommutative and commutative theories can be easily seen in the representation (4.1). Indeed, we can see that the commutative limit of (4.6) becomes (4.7). 


\section{Conclusions and Discussions}

In this paper, we showed the index theorem for the Ginsparg-Wilson Dirac operator in the 't Hooft Polyakov monopole backgrounds and provided the meaning of the projection operator. We then calculated the spectrum and eigenstates, and confirmed the index theorem by counting the number of the chiral zeromodes. We also showed that the largest-eigenvalue modes play an important role in defining the index consistently in the theories with finite degrees of freedom.

One of the mail results of the paper is that we have extended the index theorem to general configurations which are not restricted to the special type of solutions. By this generalization, configuration space can be classified into topological sectors. The commutative limit of the topological charge becomes the one introduced by 't Hooft in spontaneously broken gauge theories. We also considered the condition to assure the validity of this formulation, which gives the generalization of the admissibility conditions in the lattice gauge theory. Since this formulation is gauge invariant, it might be used to formulate the chiral gauge theory. Abelian chiral gauge theories on the lattice with exact gauge invariance was constructed by using the chiral projection operator [45]. It is an interesting future problem whether a generalization of our formulation provides an alternative to it.

It is also interesting to study the TP monopole configurations of general $\mathrm{m}$ in the commutative and noncommutative theories 13 . By some gauge transformations, TP monopole configurations can be seen as Dirac monopoles or Wu-Yang monopoles where we need to introduce the notion of patch. Hence this study will lead to a formulation of monopole bundles in the noncommutative geometries or matrix models. Also it is interesting to study whether configurations with a nontrivial index exist without introducing the projection operator. In a discretized noncommutative torus, nontrivial configurations can exist without introducing

\footnotetext{
${ }^{13}$ Some comments are given in [36].
} 
a projection operator, though their existence probability vanishes in the continuum limit [48, 49]. These studies may provide another meaning of the projection operator.

\section{Acknowledgements}

We would like to thank K. Funakubo, H. Kawai, J. Nishimura and M. Sakamoto, A. Tsuchiya for discussions and useful comments.

\section{A Spectrum of the GKP Dirac operator}

In this appendix, we will obtain the spectrum for $D_{\mathrm{GKP}}(2.7)$ in the TP monopole backgrounds (2.24):

$$
D_{\mathrm{GKP}}^{m}=\sigma_{i}\left(A_{i}-L_{i}^{R}\right)+1=\sigma_{i}\left[\left(\begin{array}{ll}
L_{i}^{(n+m)} & \\
& L_{i}^{(n-m)}
\end{array}\right)-L_{i}^{R}\right]+1
$$

Note $L_{i}^{(n \pm m)}$ has spin $L \pm m / 2$, and $-L_{i}^{R}$ has spin $L$, where $n=2 L+1$. Then the operator $A_{i}-L_{i}^{R}$ has the following spins:

$$
l=\left\{\begin{array}{lc}
\frac{m}{2}, \cdots, 2 L+\frac{m}{2} & (\delta=1), \\
\frac{m}{2}, \cdots, 2 L-\frac{m}{2} & (\delta=-1) .
\end{array}\right.
$$

Here we set $m \geq 0$. Then, by considering the spin $J$ for the operator $M_{i}$ of (3.3), we obtain the following spectrum for $D_{\mathrm{GKP}}^{m}$ :

$$
D_{\mathrm{GKP}}^{m}=\left\{\begin{array}{cccc}
J+\frac{1}{2} & = & l+1 \quad\left(J=l+\frac{1}{2}\right), \\
-\left(J+\frac{1}{2}\right) & = & -l \quad\left(J=l-\frac{1}{2}\right)
\end{array}\right.
$$

where l's are given by (A.2). The spectrum is shown in Figure 2 for $m>0$ cases. For $m=0$, the spectrum is given by Figure 2 except for the fact that zero-modes do not exist. We also illustrate the cases for $n=10, m=0,1,2,3,4$ in Figure 3 . 


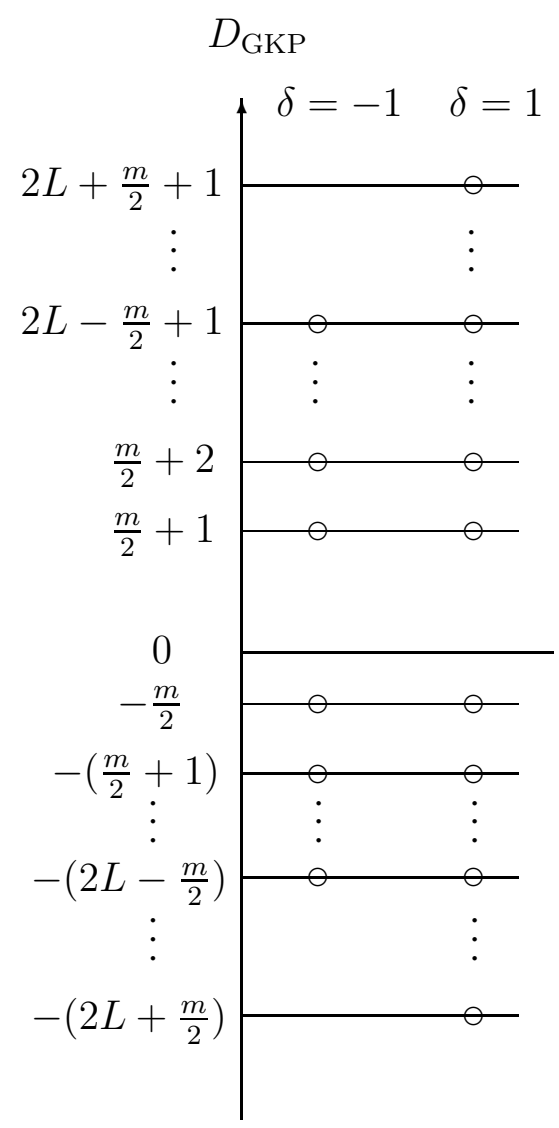

Figure 2: Spectrum for the GKP Dirac operator in the monopole backgrounds of $m>0$.

In the remainder of this appendix, we will obtain the above spectrum in another way, as we did for $D_{\mathrm{GW}}^{m}$ in section 3 . Then we can make the correspondence of the eigenstates for $D_{\text {GKP }}^{m}$ to the eigenstates in Table[1. In particular, we can see the chirality of $\Gamma^{R}, \nu$, of the $D_{\mathrm{GKP}}^{m}$ eigenstates. By straightforward calculations, we can show

$$
\left(D_{\mathrm{GKP}}^{m}\right)^{2}=M_{i}^{2}+\frac{1}{4}=J(J+1)+\frac{1}{4}=\left(J+\frac{1}{2}\right)^{2},
$$

and $\left(D_{\mathrm{GKP}}^{m}\right)^{2}$ commutes with $M_{i}, T, \Gamma^{R}$. Thus spectrum for $\left(D_{\mathrm{GKP}}^{m}\right)^{2}$ is

$$
\left(D_{\mathrm{GKP}}^{m}\right)^{2}\left|J, J_{3}, \delta, \nu\right\rangle=\left(J+\frac{1}{2}\right)^{2}\left|J, J_{3}, \delta, \nu\right\rangle
$$

where the simultaneous eigenstate $\left|J, J_{3}, \delta, \nu\right\rangle$ is defined in (3.5)-(3.8). 


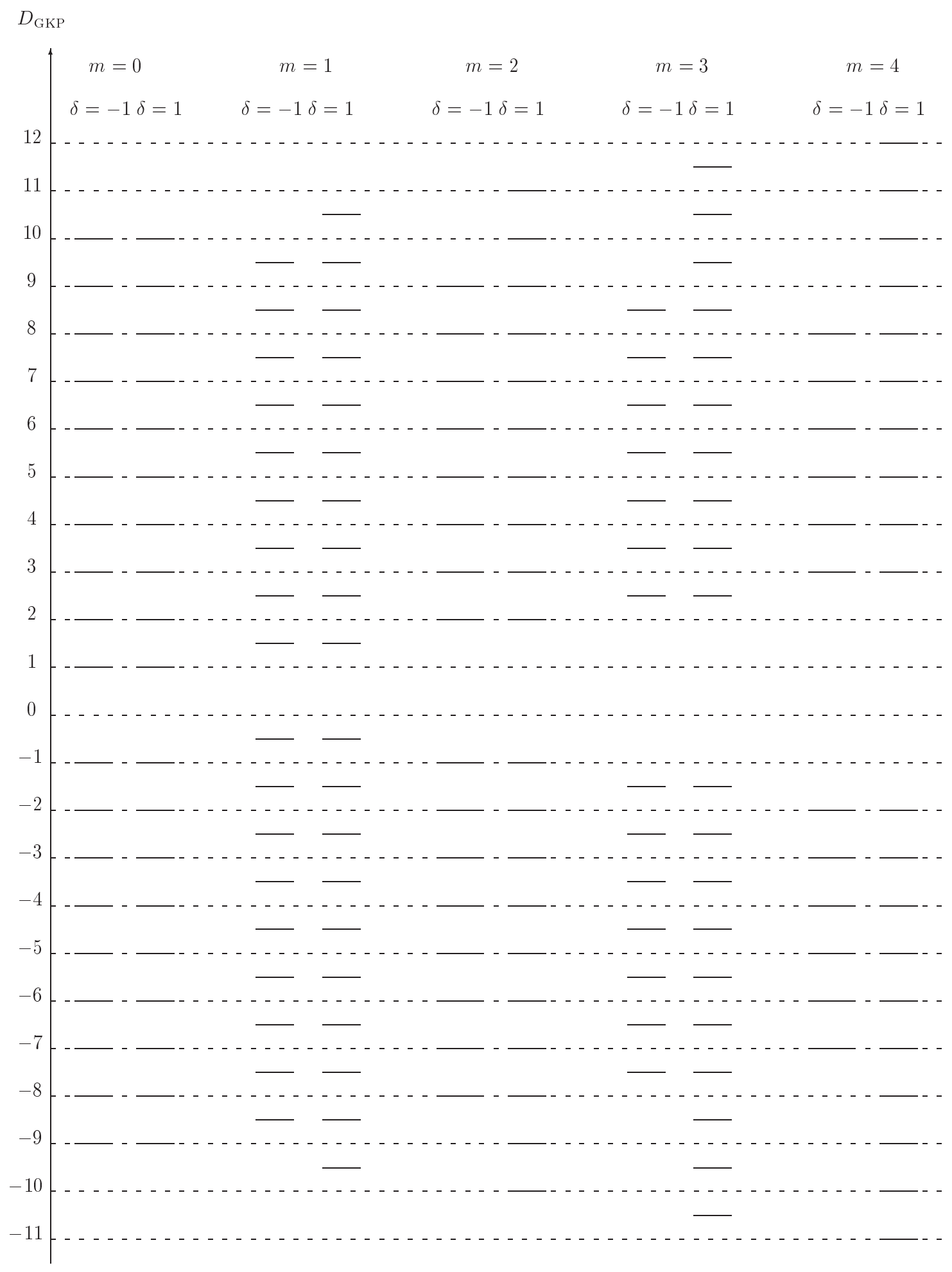

Figure 3: Spectrum for the GKP Dirac operator for the cases of $n=10$ and $m=0,1,2,3,4$. 
Since $D_{\mathrm{GKP}}^{m}$ satisfies

$$
\begin{aligned}
{\left[M_{i}, D_{\mathrm{GKP}}^{m}\right] } & =0, \\
{\left[T, D_{\mathrm{GKP}}^{m}\right] } & =0, \\
{\left[\Gamma^{R}, D_{\mathrm{GKP}}^{m}\right] } & \neq 0,
\end{aligned}
$$

linear combinations over $\nu$ for each fixed $J, J_{3}, \delta, \sum_{\nu} c_{\nu}\left|J, J_{3}, \delta, \nu\right\rangle$, give eigenstates for $D_{\mathrm{GKP}}^{m}$ as

$$
D_{\mathrm{GKP}}^{m} \sum_{\nu} c_{\nu}\left|J, J_{3}, \delta, \nu\right\rangle= \pm\left(J+\frac{1}{2}\right) \sum_{\nu} c_{\nu}\left|J, J_{3}, \delta, \nu\right\rangle .
$$

We now define

$$
D_{\mathrm{GKP}}^{m \pm}=\left(\sigma_{i} L_{i}^{(n \pm m)}+\frac{1}{2}\right)-\left(\sigma_{i} L_{i}^{R}-\frac{1}{2}\right)=\frac{n \pm m}{2} \hat{\Gamma}^{ \pm}-\frac{n}{2} \Gamma^{R},
$$

where

$$
\begin{aligned}
& \Gamma^{R}=\frac{2}{n}\left(\sigma_{i} L_{i}^{R}-\frac{1}{2}\right), \\
& \hat{\Gamma}^{ \pm}=\frac{2}{n \pm m}\left(\sigma_{i} L_{i}^{(n \pm m)}+\frac{1}{2}\right),
\end{aligned}
$$

in each of $\delta= \pm 1$ sectors. Then we can show the following Ginsparg-Wilson-like relation:

$$
\begin{aligned}
& \frac{n \pm m}{n} \hat{\Gamma}^{ \pm} D_{\mathrm{GKP}}^{m \pm}+D_{\mathrm{GKP}}^{m \pm} \Gamma^{R}= \pm m+\frac{m^{2}}{2 n}, \\
& \frac{n \pm m}{n} D_{\mathrm{GKP}}^{m \pm} \hat{\Gamma}^{ \pm}+\Gamma^{R} D_{\mathrm{GKP}}^{m \pm}= \pm m+\frac{m^{2}}{2 n} .
\end{aligned}
$$

Suppose $\psi$ is an eigenstate for $D_{\mathrm{GKP}}^{m \pm}$ with an eigenvalue $\alpha$ :

$$
D_{\text {GKP }}^{m \pm} \psi=\alpha \psi \text {. }
$$

Then, from (A.13) and (A.14),

$$
D_{\mathrm{GKP}}^{m \pm}\left(\Gamma^{R}+\frac{n \pm m}{n} \hat{\Gamma}^{ \pm}\right) \psi=-\alpha\left(\Gamma^{R}+\frac{n \pm m}{n} \hat{\Gamma}^{ \pm}\right) \psi+\left( \pm 2 m+\frac{m^{2}}{n}\right) \psi .
$$


Thus,

$$
\begin{aligned}
& D_{\mathrm{GKP}}^{m \pm}\left[\Gamma^{R}+\frac{n \pm m}{n} \hat{\Gamma}^{ \pm}-\frac{1}{2 \alpha}\left( \pm 2 m+\frac{m^{2}}{n}\right)\right] \psi \\
= & -\alpha\left[\Gamma^{R}+\frac{n \pm m}{n} \hat{\Gamma}^{ \pm}-\frac{1}{2 \alpha}\left( \pm 2 m+\frac{m^{2}}{n}\right)\right] \psi
\end{aligned}
$$

Hence, $\left[\Gamma^{R}+\frac{n \pm m}{n} \hat{\Gamma}^{ \pm}-\frac{1}{2 \alpha}\left( \pm 2 m+\frac{m^{2}}{n}\right)\right] \psi$ is an eigenstate for $D_{\mathrm{GKP}}^{m \pm}$ with an eigenvalue $-\alpha$.

From (A.10),

$$
\Gamma^{R}+\frac{n \pm m}{n} \hat{\Gamma}^{ \pm}=\frac{2}{n} D_{\mathrm{GKP}}^{m \pm}+2 \Gamma^{R} .
$$

Then, for the eigenstate $\psi=\sum_{\nu} c_{\nu}\left|J, J_{3}, \delta, \nu\right\rangle$ in (A.9),

$$
\begin{aligned}
& {\left[\Gamma^{R}+\frac{n+\delta m}{n} \hat{\Gamma}-\frac{1}{2 \alpha}\left(2 \delta m+\frac{m^{2}}{n}\right)\right] \sum_{\nu} c_{\nu}\left|J, J_{3}, \delta, \nu\right\rangle } \\
= & {\left[\frac{2}{n} \alpha-2-\frac{1}{2 \alpha}\left(2 \delta m+\frac{m^{2}}{n}\right)\right] c_{-1}\left|J, J_{3}, \delta, \nu=-1\right\rangle } \\
+ & {\left[\frac{2}{n} \alpha+2-\frac{1}{2 \alpha}\left(2 \delta m+\frac{m^{2}}{n}\right)\right] c_{1}\left|J, J_{3}, \delta, \nu=1\right\rangle, }
\end{aligned}
$$

where $\alpha= \pm\left(J+\frac{1}{2}\right)$. For the lowest spin states with $J=\frac{m-1}{2}$ in Table 1, since only $\nu=+1$ or $\nu=-1$ exists for each $\delta$, the state (A.19) must vanish. Thus we can show $\alpha=-\frac{m}{2}$. Hence only negative eigenvalue for $D_{\mathrm{GKP}}^{m}$ exist for the lowest spin states. For the highest spin states, $(J, \delta, \nu)=\left(2 L-\frac{m-1}{2},-1,-1\right)$, $\left(2 L+\frac{m+1}{2},+1,-1\right)$, only $\nu=-1$ exists for each $\delta$, and the state (A.19) must vanish. Thus we can show $\alpha=n \pm \frac{m}{2}$ for $\delta= \pm 1$. Hence only positive eigenvalues for $D_{\text {GKP }}^{m}$ exist for the highest spin states. For the other states, both positive and negative eigenvalues exit. Then we obtain the spectrum in figure 2 again.

We will also determine the coefficients $c_{\nu}$, and obtain the form of the eigenstates. Since the state (A.19) and $\sum_{\nu} c_{\nu}\left|J, J_{3}, \delta, \nu\right\rangle$ are orthogonal,

$$
\left[\frac{2}{n} \alpha-2-\frac{1}{2 \alpha}\left(2 \delta m+\frac{m^{2}}{n}\right)\right]\left|c_{-1}\right|^{2}+\left[\frac{2}{n} \alpha+2-\frac{1}{2 \alpha}\left(2 \delta m+\frac{m^{2}}{n}\right)\right]\left|c_{1}\right|^{2}=0
$$


Therefore the eigenstates for $D_{\mathrm{GKP}}^{m}$ is given by

$$
\begin{aligned}
& \frac{1}{2}\left[\sqrt{2+\frac{2}{n} \alpha-\frac{1}{2 \alpha}\left(2 \delta m+\frac{m^{2}}{n}\right)}\left|J, J_{3}, \delta, \nu=-1\right\rangle\right. \\
& \left.+\frac{\alpha}{|\alpha|} \sqrt{2-\frac{2}{n} \alpha+\frac{1}{2 \alpha}\left(2 \delta m+\frac{m^{2}}{n}\right)}\left|J, J_{3}, \delta, \nu=1\right\rangle\right]
\end{aligned}
$$

where $\alpha= \pm\left(J+\frac{1}{2}\right)$. Here we have absorbed the relative phase into the definition of $\left|J, J_{3}, \delta, \nu=-1\right\rangle$ and $\left|J, J_{3}, \delta, \nu=1\right\rangle$.

\section{B Spectrum of the Dirac operator in the com- mutative theory}

In this appendix, we will calculate the spectrum for the Ginsparg-Wilson Dirac operator in the commutative theory (2.21) in the TP monopole background (2.26) with $m=1$ :

$$
D_{\mathrm{com}}^{\prime m=1}=\sigma \cdot \mathcal{L}+1+\frac{1}{2} \sigma \cdot \tau-\frac{1}{2}(n \cdot \sigma)(n \cdot \tau) .
$$

It is also obtained by the commutative limit of (4.1).

We consider the following operators:

$$
\begin{aligned}
M_{i} & =\mathcal{L}_{i}+\frac{\sigma_{i}}{2}+\frac{\tau_{i}}{2}, \\
t & =n \cdot \tau \\
\gamma & =n \cdot \sigma
\end{aligned}
$$

where $M_{i}$ is a total angular momentum operator, $t$ is a generator for the unbroken $U(1)$ gauge group, and $\gamma$ is a chirality operator. Since they commute with one another, we can consider the simultaneous eigenstates for these operators as

$$
\begin{aligned}
M_{i}^{2}\left|J, J_{3}, \delta, \nu\right\rangle & =J(J+1)\left|J, J_{3}, \delta, \nu\right\rangle, \\
M_{3}\left|J, J_{3}, \delta, \nu\right\rangle & =J_{3}\left|J, J_{3}, \delta, \nu\right\rangle, \\
t\left|J, J_{3}, \delta, \nu\right\rangle & =\delta\left|J, J_{3}, \delta, \nu\right\rangle, \\
\gamma\left|J, J_{3}, \delta, \nu\right\rangle & =\nu\left|J, J_{3}, \delta, \nu\right\rangle .
\end{aligned}
$$


Table 2: Values of $J$ in each $\delta, \nu$ sector.

\begin{tabular}{|c|ccccc|}
\hline & $\delta$ & - & - & + & + \\
$J$ & $\nu$ & + & - & + & - \\
\hline 0 & & $\circ$ & & & $\circ$ \\
1 & & $\circ$ & $\circ$ & $\circ$ & $\circ$ \\
2 & & $\circ$ & $\circ$ & $\circ$ & $\circ$ \\
3 & & $\circ$ & $\circ$ & $\circ$ & $\circ$ \\
$\vdots$ & & $\vdots$ & $\vdots$ & $\vdots$ & $\vdots$ \\
\hline
\end{tabular}

$J$ turns out to take values as in the Table $2{ }^{14}$.

By straightforward calculations, square of $D_{\text {com }}^{\prime m=1}$ becomes

$$
\left(D_{\mathrm{com}}^{\prime m=1}\right)^{2}=M_{i}^{2}
$$

and $\left(D_{\text {com }}^{\prime m=1}\right)^{2}$ commutes with $M_{i}, t, \gamma$. We thus obtain the spectrum for $\left(D_{\text {com }}^{\prime m=1}\right)^{2}$ as follows:

$$
\left(D_{\mathrm{com}}^{\prime m=1}\right)^{2}\left|J, J_{3}, \delta, \nu\right\rangle=J(J+1)\left|J, J_{3}, \delta, \nu\right\rangle
$$

Note that the states with the lowest spin, $J=0$, in figure 2 correspond to the zero-modes for $D_{\text {com }}^{\prime m}$.

We can also show

$$
\begin{aligned}
{\left[M_{i}, D_{\mathrm{com}}^{\prime m=1}\right] } & =0, \\
{\left[t, D_{\mathrm{com}}^{\prime m=1}\right] } & =0, \\
{\left[\gamma, D_{\mathrm{com}}^{\prime m=1}\right] } & \neq 0 .
\end{aligned}
$$

\footnotetext{
14 As we will see later in (B.19), two zero-modes exist as $(\delta, \nu)=(-1,+1)$ and $(+1,-1)$. From (B.11), (B.12), (B.15), the eigenstates with both $\nu=+1$ and $\nu=-1$ exist in each $J, J_{3}, \delta$ for $J \geq 1$. (Also, by defining $D^{\prime}=\tau \cdot \mathcal{L}+1+\frac{1}{2} \sigma \cdot \tau-\frac{1}{2}(n \cdot \sigma)(n \cdot \tau)$, we can show that the eigenstates with both $\delta=+1$ and $\delta=-1$ exist in each $J, J_{3}, \nu$ for $J \geq 1$.) We can also show that there is no degeneracy in each $\left|J, J_{3}, \delta, \nu\right\rangle$, by counting the number of states in each $J$.
} 
Then, linear combinations over $\nu$ for each $J, J_{3}, \delta, \sum_{\nu} c_{\nu}\left|J, J_{3}, \delta, \nu\right\rangle$, give eigenstates for the Dirac operator $D_{\text {com }}^{\prime m=1}$ as

$$
D_{\mathrm{com}}^{\prime m=1}\left[\sum_{\nu} c_{\nu}\left|J, J_{3}, \delta, \nu\right\rangle\right]= \pm \sqrt{J(J+1)}\left[\sum_{\nu} c_{\nu}\left|J, J_{3}, \delta, \nu\right\rangle\right] .
$$

Since Dirac operator $D_{\text {com }}^{\prime m=1}$ anti-commutes with the chirality operator $\gamma$ :

$$
\left\{\gamma, D_{\text {com }}^{\prime m=1}\right\}=0
$$

if $\sum_{\nu} c_{\nu}\left|J, J_{3}, \delta, \nu\right\rangle$ is an eigenstate for the $D_{\text {com }}^{\prime m=1}$ with eigenvalue $\alpha, \gamma \sum_{\nu} c_{\nu}\left|J, J_{3}, \delta, \nu\right\rangle$ is an eigenstate with eigenvalue $-\alpha$. Since

$$
\gamma \sum_{\nu} c_{\nu}\left|J, J_{3}, \delta, \nu\right\rangle=-c_{-1}\left|J, J_{3}, \delta, \nu=-1\right\rangle+c_{1}\left|J, J_{3}, \delta, \nu=1\right\rangle
$$

does not vanish, both positive and negative eigenvalues for $D_{\text {com }}^{\prime m=1}$ exist in each $J \geq 1$ and $\delta$.

We next determine the coefficients $c_{\nu}$, and obtain the form of the eigenstates. Since the state (B.16) is orthogonal to $\sum_{\nu} c_{\nu}\left|J, J_{3}, \delta, \nu\right\rangle$,

$$
\left|c_{1}\right|^{2}-\left|c_{-1}\right|^{2}=0
$$

Therefore, the eigenstates for the eigenvalue $\alpha$ are

$$
\frac{1}{\sqrt{2}}\left[\left|J, J_{3}, \delta, 1\right\rangle+\frac{\alpha}{|\alpha|}\left|J, J_{3}, \delta,-1\right\rangle\right],
$$

where $\alpha= \pm \sqrt{J(J+1)}$. Here we have absorbed the relative phase into the definition of $\left|J, J_{3}, \delta, 1\right\rangle$ and $\left|J, J_{3}, \delta,-1\right\rangle$.

Finally, we obtain configuration form of the chiral zero-modes. Since they correspond to the zero-modes of $M_{i}$, the modes with $J=0$ in Table 2 , they can be written as $\epsilon_{\alpha l}$ and $n^{i} \sigma_{\alpha \alpha^{\prime}}^{i} \epsilon_{\alpha^{\prime} l}$, where $\alpha$ and $l$ are spinor and gauge group indices respectively. We can indeed show $\left(D_{\text {com }}^{\prime m=1}\right) \epsilon=0,\left(D_{\text {com }}^{\prime m=1}\right) n \cdot \sigma \epsilon=0$ directly. Chiral zero-modes can be obtained by the linear combinations of these two zero-modes. The states with $(\delta, \nu)= \pm(-1,+1)$ are

$$
\frac{1}{2}[1 \pm n \cdot \sigma]_{\alpha \alpha^{\prime}} \epsilon_{\alpha^{\prime} l}=\frac{1}{2}[1 \mp n \cdot \tau]_{l l^{\prime}} \epsilon_{\alpha l^{\prime}},
$$


where we used the identity (4.3).

The eigenvalues and eigenstates obtained here agree with the commutative limit of the results in section 3 and section 4 . We also note that the monopole harmonics in the commutative theory was provided in [50], and the spectrum of the equivalent Dirac operator was studied in [51].

\section{References}

[1] T. Banks, W. Fischler, S. H. Shenker and L. Susskind, Phys. Rev. D 55, 5112 (1997) arXiv:hep-th/9610043.

[2] N. Ishibashi, H. Kawai, Y. Kitazawa and A. Tsuchiya, Nucl. Phys. B 498, 467 (1997) arXiv:hep-th/9612115.

For a review, see H. Aoki, S. Iso, H. Kawai, Y. Kitazawa, A. Tsuchiya and T. Tada, Prog. Theor. Phys. Suppl. 134, 47 (1999) arXiv:hep-th/9908038.

[3] A. Connes, Noncommutative geometry, Academic Press, 1990.

[4] A. Connes, M. R. Douglas and A. Schwarz, JHEP 9802, 003 (1998) arXiv:hep-th/9711162|.

[5] H. Aoki, N. Ishibashi, S. Iso, H. Kawai, Y. Kitazawa and T. Tada, Nucl. Phys. B 565, 176 (2000) arXiv:hep-th/9908141.

[6] N. Seiberg and E. Witten, JHEP 9909 (1999) 032 arXiv:hep-th/9908142.

[7] H. Grosse, C. Klimcik and P. Presnajder, Commun. Math. Phys. 178, 507 (1996) arXiv:hep-th/9510083;

S. Baez, A. P. Balachandran, B. Ydri and S. Vaidya, Commun. Math. Phys. 208, 787 (2000) arXiv:hep-th/9811169;

G. Landi, J. Geom. Phys. 37, 47 (2001) arXiv:math-ph/9905014. 
[8] A. P. Balachandran and S. Vaidya, Int. J. Mod. Phys. A 16, 17 (2001) arXiv:hep-th/9910129.

[9] P. Valtancoli, Mod. Phys. Lett. A 16, 639 (2001) arXiv:hep-th/0101189.

[10] H. Steinacker, Nucl. Phys. B 679, 66 (2004) arXiv:hep-th/0307075.

[11] D. Karabali, V. P. Nair and A. P. Polychronakos, Nucl. Phys. B 627, 565 (2002) arXiv:hep-th/0111249.

[12] U. Carow-Watamura, H. Steinacker and S. Watamura, J. Geom. Phys. 54, 373 (2005) arXiv:hep-th/0404130.

[13] P. H. Ginsparg and K. G. Wilson, Phys. Rev. D 25, 2649 (1982).

[14] H. Neuberger, Phys. Lett. B 417, 141 (1998) arXiv:hep-lat/9707022]; Phys. Rev. D 57, 5417 (1998) arXiv:hep-lat/9710089]; Phys. Lett. B 427, 353 (1998) arXiv:hep-lat/9801031.

[15] P. Hasenfratz, Nucl. Phys. Proc. Suppl. 63, $53 \quad$ (1998) arXiv:hep-lat/9709110; P. Hasenfratz, V. Laliena and F. Niedermayer, Phys. Lett. B 427, 125 (1998) arXiv:hep-lat/9801021.

[16] M. Lüscher, Phys. Lett. B 428, 342 (1998) [arXiv:hep-lat/9802011].

[17] F. Niedermayer, Nucl. Phys. Proc. Suppl. 73, $105 \quad$ (1999) arXiv:hep-lat/9810026.

[18] H. Aoki, S. Iso and K. Nagao, Phys. Rev. D 67, 085005 (2003) arXiv:hep-th/0209223.

[19] J. Madore, Class. Quant. Grav. 9, 69 (1992).

[20] A. P. Balachandran, T. R. Govindarajan and B. Ydri, Mod. Phys. Lett. A 15, 1279 (2000) arXiv:hep-th/9911087); arXiv:hep-th/0006216. 
[21] B. Ydri, JHEP 0308, 046 (2003) arXiv:hep-th/0211209.

[22] A. P. Balachandran and G. Immirzi, Phys. Rev. D 68, 065023 (2003) arXiv:hep-th/0301242].

[23] H. Aoki, S. Iso and K. Nagao, Nucl. Phys. B 684, 162 (2004) arXiv:hep-th/0312199.

[24] J. Nishimura and M. A. Vazquez-Mozo, JHEP 0108, 033 (2001) arXiv:hep-th/0107110].

[25] S. Iso and K. Nagao, Prog. Theor. Phys. 109, 1017 (2003) arXiv:hep-th/0212284.

[26] T. Fujiwara, K. Nagao and H. Suzuki, JHEP 0209, 025 (2002) arXiv:hep-lat/0208057].

[27] J. Nishimura and M. A. Vazquez-Mozo, JHEP 0301, 075 (2003) arXiv:hep-lat/0210017.

[28] H. Aoki, S. Iso, T. Maeda and K. Nagao, Phys. Rev. D 71, 045017 (2005) arXiv:hep-th/0412052

[29] R. C. Myers, JHEP 9912 (1999) 022 [arXiv:hep-th/9910053].

[30] S. Iso, Y. Kimura, K. Tanaka and K. Wakatsuki, Nucl. Phys. B 604, 121 (2001) arXiv:hep-th/0101102.

[31] S. Bal and H. Takata, Int. J. Mod. Phys. A 17, 2445 (2002) arXiv:hep-th/0108002.

[32] P. Valtancoli, Int. J. Mod. Phys. A 18, 967 (2003) arXiv:hep-th/0206075].

[33] T. A. Imai, Y. Kitazawa, Y. Takayama and D. Tomino, Nucl. Phys. B 665, 520 (2003) arXiv:hep-th/0303120]. 
[34] T. Azuma, S. Bal, K. Nagao and J. Nishimura, JHEP 0405, 005 (2004) arXiv:hep-th/0401038; JHEP 0605, 061 (2006) arXiv:hep-th/0405277); T. Azuma, K. Nagao and J. Nishimura, JHEP 0506, 081 (2005) arXiv:hep-th/0410263.

[35] P. Castro-Villarreal, R. Delgadillo-Blando and B. Ydri, Nucl. Phys. B 704, 111 (2005) arXiv:hep-th/0405201.

[36] G. Ishiki, S. Shimasaki, Y. Takayama and A. Tsuchiya, JHEP 0611, 089 (2006) arXiv:hep-th/0610038.

[37] U. Carow-Watamura and S. Watamura, Commun. Math. Phys. 183, 365 (1997) arXiv:hep-th/9605003; Commun. Math. Phys. 212, 395 (2000) arXiv:hep-th/9801195.

[38] H. Grosse and J. Madore, Phys. Lett. B 283, 218 (1992);

H. Grosse and P. Presnajder, Lett. Math. Phys. 33, 171 (1995);

H. Grosse, C. Klimcik and P. Presnajder, Commun. Math. Phys. 185, 155 (1997) arXiv:hep-th/9507074; arXiv:hep-th/9603071.

[39] H. Grosse and P. Presnajder, arXiv:hep-th/9805085; Lett. Math. Phys. 46, 61 (1998).

[40] P. Presnajder, J. Math. Phys. 41, 2789 (2000) arXiv:hep-th/9912050.

[41] H. Aoki, S. Iso and K. Nagao, Phys. Rev. D 67, 065018 (2003) arXiv:hep-th/0209137.

[42] G. 't Hooft, Nucl. Phys. B 79, 276 (1974).

[43] M. Luscher, Commun. Math. Phys. 85, 39 (1982).

[44] P. Hernandez, K. Jansen and M. Luscher, Nucl. Phys. B 552, 363 (1999) arXiv:hep-lat/9808010. 
[45] M. Luscher, Nucl. Phys. B 538, 515 (1999) arXiv:hep-lat/9808021; Nucl. Phys. B 549, 295 (1999) arXiv:hep-lat/9811032.

[46] H.Aoki and S.Iso, in preparation

[47] K. Fujikawa, Phys. Rev. D 60, 074505 (1999) arXiv:hep-lat/9904007|.

[48] K. Nagao, Phys. Rev. D 73, 065002 (2006) arXiv:hep-th/0509034].

[49] H. Aoki, J. Nishimura and Y. Susaki, JHEP 0702, 033 (2007) arXiv:hep-th/0602078; arXiv:hep-th/0604093.

[50] T. T. Wu and C. N. Yang, Nucl. Phys. B 107, 365 (1976).

[51] V. A. Rubakov, Nucl. Phys. B 203, 311 (1982). 